\title{
BMJ Open CARE CR-Cardiovascular and \\ cardiorespiratory Adaptations to Routine Exercise-based Cardiac Rehabilitation: a study protocol for a community-based controlled study with criterion methods
}

\author{
Simon Nichols, ${ }^{1}$ Fiona Nation, ${ }^{2}$ Toni Goodman, ${ }^{3}$ Andrew L Clark, ${ }^{4}$ Sean Carroll, ${ }^{2}$ \\ Lee Ingle ${ }^{2}$
}

To cite: Nichols S, Nation F, Goodman T, et al. CARE CR-Cardiovascular and cardiorespiratory Adaptations to Routine Exercise-based Cardiac Rehabilitation: a study protocol for a communitybased controlled study with criterion methods. BMJ Open 2018;8:e019216. doi:10.1136/ bmjopen-2017-019216

- Prepublication history for this paper is available online. To view these files, please visit the journal online (http://dx.doi org/10.1136/bmjopen-2017019216).

Received 18 August 2017 Revised 25 0ctober 2017 Accepted 22 November 2017

Check for updates

${ }^{1}$ Centre for Sport and Exercise Science, Sheffield Hallam University-Collegiate Crescent Campus, Sheffield, UK

${ }^{2}$ Department of Sport Health and Exercise Science, University of Hull, Hull, UK

${ }^{3}$ Integrated Community Services, City Health Care Partnership CIC, East Riding Community Hospital, Beverley, UK

${ }^{4}$ Department of Academic

Cardiology, Castle Hill Hospital, Cottingham, UK

Correspondence to

Dr Simon Nichols;

s.j.nichols@shu.ac.uk

\section{ABSTRACT}

Introduction Cardiac rehabilitation (CR) reduces all-cause and cardiovascular mortality in patients with coronary heart disease (CHD). Much of this improvement has been attributed to the beneficial effects of structured exercise training. However, UK-based studies have not confirmed this. Improvements in survival and cardiovascular health are associated with concurrent improvements in cardiorespiratory fitness (CRF). It is therefore concerning that estimated CRF improvements resulting from UKbased CR are approximately one-third of those reported in international literature. Modest improvements in CRF suggest that UK CR exercise training programmes may require optimisation if long-term survival is to be improved. However, contemporary UK studies lack control data or use estimates of CRF change. Cardiovascular and cardiorespiratory Adaptations to Routine Exercisebased CR is a longitudinal, observational, controlled study designed to assess the short-term and long-term effect of CR on CRF, as well cardiovascular and cardiometabolic health.

Methods and analysis Patients will be recruited following referral to their local CR programme and will either participate in a routine, low-to-moderate intensity, 8-week (16 sessions) exercise-based CR programme or freely abstain from supervised exercise. Initial assessment will be conducted prior to exercise training, or approximately 2 weeks after referral to $C R$ if exercise training is declined. Reassessment will coincide with completion of exercise training or 10 weeks after initial assessment for control participants. Participants will receive a final follow-up 12 months after recruitment. The primary outcome will be peak oxygen consumption determined using maximal cardiopulmonary exercise testing. Secondary outcomes will include changes in subclinical atherosclerosis (carotid intima-media thickness and plaque characteristics), body composition (dual X-ray absorptiometry) and cardiometabolic biomarkers.

Ethics and dissemination Ethical approval for this non-randomised controlled study has been obtained from the Humber Bridge NHS Research Ethics Committee-

\section{Strengths and limitations of this study}

- The use of 'gold-standard' maximal cardiopulmonary exercise testing will provide some of the most accurate and objective cardiorespiratory fitness outcomes derived from UK cardiac rehabilitation (CR) data.

- Carotid intima-media thickness measurements will demonstrate the effect of $\mathrm{CR}$ on atherosclerotic disease progression.

- The observational nature of this study conducted within local CR ensures ecological validity of our findings.

- The non-randomised nature of this study may result in group allocation bias.

- This is a single-centre study with participant referral/ recruitment constraints that are characteristic of exercise training within UK-based CR.

Yorkshire and the Humber on the 27th September 2013, $(12 / \mathrm{YH} / 0278)$. Results will be presented at national conferences and published in peer-reviewed journals.

\section{INTRODUCTION}

Coronary heart disease (CHD) affects 2.3 million people in the UK and is a leading cause of premature death. ${ }^{1}$ Improvements in diagnosis and medical treatment have resulted in improved survival rates, however, the burden of CHD remains a major public health challenge. Cardiac rehabilitation (CR) is a comprehensive programme of secondary prevention measures that has been shown to have significant health benefits for patients with CHD.

The aim of CR is to increase survival, reduce cardiovascular disease (CVD)-related morbidity and hospital admissions, improve 
functional capacity, quality of life and facilitate early return to work. ${ }^{2}{ }^{3}$ This is achieved through structured exercise training and increasing physical activity, preventive medical therapies, education and behaviour change, counselling support and other cardiovascular risk factor reduction strategies. ${ }^{2}{ }^{4}$ Although variations in service provision exist across the $\mathrm{UK},{ }^{5}$ CR exercise training is usually offered in the early postadmission period following a cardiac event. The UK healthcare system no longer uses 'Phases' to describe CR, however, early postadmission supervised exercise training may be equated to Phase III CR.

Structured exercise training is one of the primary components of $\mathrm{CR}^{267}$ and may make the largest contribution to increasing patient survival. ${ }^{89}$ Exercise training alone is associated with a $28 \%$ all-cause mortality reduction. ${ }^{10}$ Contemporary evidence suggests that all-cause and CVD mortality, recurrent cardiac events, ${ }^{11}$ and hospital admissions are reduced while quality of life is improved. ${ }^{9}$ However, a recent Cochrane review questioned these findings and reported that CVD mortality $(10.4 \%$ to $7.6 \%)$ but not all-cause mortality was reduced following CR. ${ }^{9}{ }^{12}$

Contradictory to consecutive meta-analyses, ${ }^{9} 11 \quad 13$ UK-derived data suggest that CR may not improve CVD or all-cause mortality. ${ }^{14-16}$ The most recent UK randomised controlled study reported no survival benefit, ${ }^{16}$ though did not consider cardiorespiratory fitness (CRF) changes. Peak oxygen uptake $\left(\mathrm{VO}_{2 \text { peak }}\right.$ (determined during maximal cardiopulmonary exercise testing $(\mathrm{CPET})^{17}$ is used to quantify CRF. $\mathrm{VO}_{2 \text { peak }}$ is inversely associated with all-cause and cardiovascular mortality in patients with CHD. ${ }^{18} 19$ A $1 \%$ improvement in $\mathrm{VO}_{2 \text { peak }}$ following 3 months exercise training confers a $2 \%$ reduction in cardiovascular mortality ${ }^{20}$ with the least fit patients showing the greatest survival advantage from any improvements. ${ }^{22}$ However, a dose-response relationship between the amount of exercise training undertaken and increase in $\mathrm{VO}_{2 \text { peak }}$ may exist. ${ }^{20}$

UK clinical trial data ${ }^{23}$ in patients who sustained a myocardial infarction (MI), reported increases in $\mathrm{VO}_{\text {2peak }}$ following 12 months supervised exercise training compared with controls. However, a recent multicentre study of routine UK-based CR (current clinical practice) indicates that the 'exercise dose' within outpatient CR may be insufficient to meaningfully improve $\mathrm{CRF}^{24} 25$ ( 0.5 metabolic equivalents [METs]; or $\mathrm{VO}_{2} 1.75 \mathrm{~mL} / \mathrm{kg} / \mathrm{min}$ ) when compared with international programmes ( 1.5 METs; or $\mathrm{VO}_{2} 5.25 \mathrm{~mL} /$ $\mathrm{kg} / \mathrm{min}) .{ }^{26}$ Fewer than $50 \%$ of patients completing a 'typical' UK CR programme may achieve minimal clinically important improvements to CRF, $(70 \mathrm{~m})$ derived from incremental shuttle walk testing. ${ }^{27}$ These findings may explain why UK CR programmes do not appear to improve patient survival. ${ }^{14-16}$ However, UK studies typically estimate CRF changes from submaximal exercise testing protocols. This may lead to inaccurate reporting of $\mathrm{VO}_{\text {2peak }}$ changes following $\mathrm{CR}$ in patients with CHD. ${ }^{28}$ There is a need to investigate the exercise-based CR findings of Sandercock et $a l^{29}$ using 'gold-standard' CPET testing methods.

Numerous mechanisms may be responsible for improving survival associated with exercise-based CR and improved CRF, including cardiovascular risk factor modification (smoking, lipids, blood pressure (BP), glucose metabolism). Within one meta-analysis, approximately half of the $28 \%$ reduction in cardiac mortality achieved with exercise-based CR was attributed to reductions in major cardiovascular risk factors, particularly reduced smoking. ${ }^{9}$ Anti-ischaemic/thrombotic effects, cardiac remodelling, and antiatherosclerotic and vascular conditioning have also been documented. ${ }^{30} 31$ Larger volumes of exercise training (associated with higher energy expenditures) have been shown to underline regression of atherosclerosis. ${ }^{32}$ Carotid intima-media thickness (C-IMT) is a practical, valid and reliable non-invasive surrogate marker of subclinical atherosclerosis. ${ }^{33-35}$ Carotid ultrasound has been used to non-invasively characterise dynamic changes in atherosclerotic plaque characteristics. While some data suggest that exercise training may reduce C-IMT in patients at elevated CV risk, ${ }^{36}{ }^{37}$ the evidence is still unclear. ${ }^{38}$ Furthermore, no UK study has investigated the effects of a short-term, routine CR exercise training programme on longer-term atherosclerotic disease progression. The modest improvements in CRF reported within UK CR patients, ${ }^{29} 39$ and the reported absence of improved survival outcomes, may indicate that the exercise dose prescribed to patients is too low to meaningfully influence CRF, cardiometabolic risk factors and atherosclerotic plaque progression. Therefore, the objectives of this controlled trial are:

To determine, when compared with CR without exercise training, the short-term (8-week) and long-term (12-month) effects of a routine, 8 week, low-to-moderate intensity UK CR exercise training programme on:

1. Changes in $\mathrm{VO}_{2 \text { peak }}$ assessed using 'gold-standard' CPET.

2. Subclinical and clinical atherosclerosis progression using C-IMT measurements.

3. Standard risk factors including lipid profiles, BP and blood glucose, measurement, and cardiometabolic markers including $\mathrm{N}$-terminal pro-brain natriuretic peptide (NT-proBNP) and high-sensitivity C-reactive protein (hs-CRP).

4. Estimated all-cause 5-year mortality risk using the comprehensive CALIBER score. ${ }^{40}$

\section{METHODS}

\section{Study design}

This study will be a pragmatic, single-centre longitudinal controlled study of a routine National Health Service (NHS) outpatient CR programme. Patients recruited to the study will have the option to attend a routine low-to-moderate intensity, 8-week circuit-based CR exercise training programme (routine CR) or voluntarily abstain (control group (CG)) from the structured 
exercise training component of the CR programme. Study measures will be made before starting exercise training or approximately 2 weeks after recruitment for patients who decline the exercise programme (visit 1). Follow-up assessment will be conducted after completion of a patients CR programme (visit 2) or approximately 10 weeks after recruitment for controls. The difference in planned reassessment times accounts for a typical 2-week waiting time to receive NHS treatment (exercise training) at this centre, and will allow both groups to be reassessed within a similar timeframe. Patients will also be invited for assessment 12 months after visit 1 (visit 3 ).

Routine CR will be delivered by clinical (not research) staff within existing NHS secondary prevention care pathways. The study will be conducted in collaboration with Hull's CR team (City Healthcare Partnership CIC) who follow the Department of Health ${ }^{41}$ 'best care pathway' for referral and delivery of CR. Adherence to national guidelines on exercise prescription will allow broad generalisability of the findings to UK-based CR programmes. The trial protocol adheres to the Standard Protocol Items: Recommendations for Clinical Trials guidelines.

\section{Setting}

Patients can attend CR at three sites across Hull: The University of Hull (West Hull), Hull Royal Infirmary (Hull Centre) and the Freedom Centre (Community Centre, East Hull). Testing will be conducted at the Academic Cardiology Research Laboratory at Castle Hill Hospital, Hull.

\section{Participants}

Patients who have had a recent hospital admission for stable angina, MI (ST-Elevation or non ST-elevationMI and non-STEMI), coronary artery bypass graft (CABG) surgery, and elective percutaneous coronary intervention (PCI) will be recruited recruitment by a specialist CR nurse, typically within 2 weeks of sustaining a cardiac event. Patients will be offered all CR secondary prevention components recommended by the British Association for Cardiovascular Prevention and Rehabilitation (BACPR), ${ }^{2}$ including exercise training. Those opting to take part in structured, supervised exercise training will be referred to as the treatment group (TG). Those who decline exercise training will be known as the control group (CG). Group randomisation will not be performed as this is deemed unethical given the current evidence for the benefits of exercise-based CR. ${ }^{9}$ Patients in both groups will be advised to increase unsupervised physical activity levels.

\section{General inclusion criteria}

1. Primary diagnosis of CHD including recent MI, CABG surgery, elective PCI or exertional angina.

2. Clinically stable patients.

3. Aged $30-85$ years.

4. Absence of contraindications to exercise testing and exercise training.
5. Capable and mentally able to understand and follow the instructions of the health professional team.

\section{General exclusion criteria}

1. Clinically unstable patients.

2. Clinically significant valvular heart disease.

3. Patients with a non-ischaemic diagnosis.

4. Patients with coexisting congenital heart conditions, significant comorbidities including severe chronic heart failure (left ventricular ejection fraction (LVEF) $<30 \%$ ), advanced cancer and conditions preventing the patient from providing informed consent.

5. Current drug abusers and excessive alcohol drinkers.

6. Patients not freely living in the community, such as those currently serving a custodial sentence

7. Patients unwilling or unable to participate in key aspects of the study.

8. Patients with ongoing clinical complications, open wounds or systemic infections.

9. Women who are pregnant or breast feeding.

A study flow diagram is presented in figure 1. Patients will be referred to CR via the local tertiary hospital (Castle Hill Hospital, Hull) where they will receive a one-to-one assessment with a CR specialist nurse. Nursing staff will provide patients with information on cardiac medications, diet, smoking cessation, physical activity, structured exercise training and other secondary prevention measures. Eligible patients will be offered the opportunity to participate in this study. Group specific patient information sheets will be provided.

Written informed consent will be obtained by a medical doctor at the Academic Cardiology Research Laboratory, Castle Hill Hospital, Hull. Patients will be asked to attend in a euhydrated state and having not conducted strenuous exercise within the previous 24 hours. Patients will not fast prior to any visit due to the need to conduct maximal CPET at the end of the 4-hour visit. Patients will be advised to eat a light meal prior to each visit.

A resting ECG, echocardiogram, venepuncture, carotid ultrasound (C-IMT) and dual X-ray absorptiometry (DXA) will be performed at each visit. A CPET to volitional exhaustion or clinically relevant symptoms ${ }^{42}$ will be conducted after all other investigations have been completed. Patients will then follow their chosen treatment plan (treatment or control). All measurements taken at visit 1 will be repeated at visit 2 and 3 . At visit 2 and 3 , all patients will be asked to verbally report the typical number of structured exercise sessions they engaged in during the previous week, as well as how many minutes each of those session lasted. This will allow a comparison of exercise dose between both groups. Adverse events will be reported in accordance with NHS good clinical practice guidelines.

\section{Anthropometry and resting haemodynamic measurements}

Patients will be instructed to remove footwear, jackets and items from their pockets prior to standing in the centre 


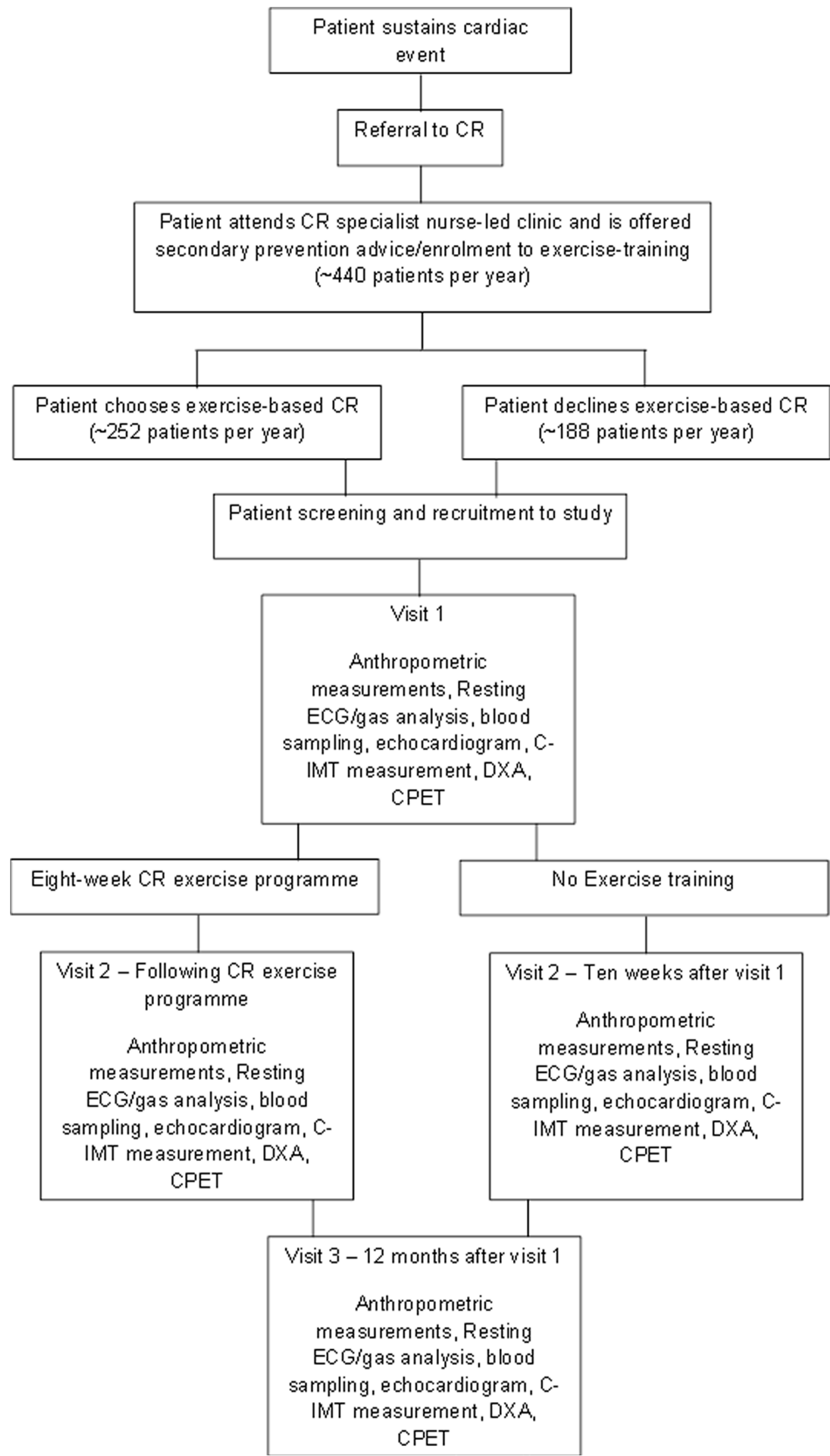

Figure 1 Study flow diagram. C-IMT, carotid intima-media thickness; CPET, cardiopulmonary exercise testing; CR, cardiac rehabilitation; DXA, dual X-ray absorptiometry. 
of the scales. Body mass (kilograms) will be measured using a Tanita Body Composition Analyser MC-180 MA (Tanita, Amsterdam, The Netherlands) and recorded to one decimal place. Stature (centimetre) will be measured (Leicester Height Measure, SECA, Birmingham, UK) with patients positioned in the Frankfort plane and their heels and head positioned to the back of the stadiometer. The highest measurement recorded during a single full in-breath will be taken as the individual's height. Body mass index (BMI) will reported as $\mathrm{kg} \cdot \mathrm{m}^{2}$, where $\mathrm{kg}$ is a patients' body mass and $\mathrm{m}^{2}$ is height squared.

A single waist and hip circumference measurements will be taken $1 \mathrm{~cm}$ above the iliac crest, and from the widest aspect of the buttocks using an inflexible tape. Both measurements will be recorded in centimetre and the waist-to-hip circumference ratio (waist/hip) will be reported. ${ }^{43}$

Patients will rest for $15 \mathrm{~min}$ in a semisupine position on an examination bed. A 12-lead ECG (GE Healthcare, Buckinghamshire, UK) and left arm brachial BP will be recorded using an ECG-gated automated BP cuff (Tango, SunTech Medical, Eynsham, UK). Resting HR and BP will be recorded following the 15-min rest period.

\section{Cardiopulmonary exercise testing}

Respiratory gas exchange data will be collected using an Oxycon Pro (Jaeger, Hochberg, Germany) breath-bybreath metabolic cart. Calibration to ambient temperature, humidity, altitude and barometric pressure will be performed. Gas flow-volume will be calibrated using a $3 \mathrm{~L}$ syringe and will be repeated on at least two occasions. Offset values are automatically calculated for accurate measurement of ventilatory volumes. Two-point calibration, using known gas concentrations, will be performed to allow accurate quantification of inspired $\mathrm{O}_{2}$ and expired $\mathrm{CO}_{2}$ concentrations (control gases: $\mathrm{O}_{2} 16.4 \%$; $\left.\mathrm{CO}_{2} 4.5 \%\right)$. The 12-lead ECG will be measured continuously throughout the CPET. An ECG-gated automated BP will be monitored from the start of CPET and at the second minute of each exercise test stage until the end of the test.

CPET will be conducted according to international recommendations. ${ }^{42}{ }^{44-46} \mathrm{~A}$ description of the CPET protocol, Borg's rating of percieved exersion (RPE) scale, potential adverse symptoms and CPET stop

\begin{tabular}{llc}
\hline Table 1 & The modified Bruce protocol & \\
\hline Stage & Speed $(\mathbf{m p h})$ & Gradient $(\%)$ \\
\hline 0 & 1.7 & 0 \\
1 & 1.7 & 5 \\
2 & 1.7 & 10 \\
3 & 2.5 & 12 \\
4 & 3.4 & 14 \\
5 & 4.2 & 16 \\
\hline 6 & 5.0 & 18 \\
\hline
\end{tabular}

procedures will be given to participants. The modified Bruce protocol ${ }^{47}$ will be used for all CPETs (table 1).

Exercise tests will be preceded by a 3-min seated rest period to record pretest gas exchange, $\mathrm{BP}$ and $\mathrm{HR}$ values. Patients will undertake CPET on a treadmill (General Electric driven by a GE case system (GE Healthcare, Buckinghamshire, UK). Ventilatory expired gases will be collected continuously during the rest period, exercise and a 6-min recovery period. Talking during CPET will be discouraged with the exception of reporting symptoms, asking to stop exercise and to provide serial RPE scores.

HR, RPE and estimated arterial oxygen saturation $\left(\mathrm{SpO}_{2}\right)$ will be obtained after $2.5 \mathrm{~min}$ of each test stage, at peak exercise and during the recovery period. Criteria for termination for CPET are displayed in box $1 .^{40}$

Data will be saved and exported for offline analysis. Data will be exported in $30 \mathrm{~s}, 15 \mathrm{~s}$ and middle 5 of 7 , breath-bybreath averages. Table 2 provides a list of traditional and novel CPET variables.

The primary outcome measure will be the change in $\mathrm{VO}_{2 \text { peak }}$ (mean $\mathrm{VO}_{2}$ over final $30 \mathrm{~s}$ of a CPET). Secondary CRF outcome measures, including the ventilatory anaerobic threshold (VAT), VE/ $/ \mathrm{VCO}_{2}$ slope, peak $\mathrm{O}_{2}$ pulse $\left(\mathrm{O}_{2} / \mathrm{HR}\right), \mathrm{O}_{2}$ uptake efficiency slope and, $\mathrm{O}_{2}$ uptake efficiency plateau will be assessed.

\section{Spirometry}

Resting spirometry will be conducted using an Oxycon Pro. Patients will breathe into a mouth piece connected to the respiratory flow turbine of the metabolic cart. Patients will be instructed to breathe normally during resting tidal volume measurements (litres). Ten full breathing cycles will be observed to allow normalisation of the breathing pattern. Flow-volume loops will be conducted to obtain forced spirometry measurements. Demonstration and instruction will be given prior to patients attempting the manoeuvre. Up to eight flow-volume loops will be conducted to obtain three high-quality manoeuvres. Acceptable reproducibility will be defined as $\leq 0.150 \mathrm{~L}$ difference between the largest and second largest forced

\section{Box 1 Exercise test termination criteria}

- Indications for exercise test termination

- Chest pain suggestive ischaemia

- Ischaemic ECG changes (>2 mm ST segment depression)

- Complex ventricular ectopy

- Second or third degree heart block

- Fall in systolic pressure $20 \mathrm{~mm} \mathrm{Hg}$ from highest value during the test

- Hypertension ( $250 \mathrm{~mm} \mathrm{Hg}$ systolic; $120 \mathrm{~mm} \mathrm{Hg}$ diastolic)

- Severe oxygen desaturation: $\mathrm{SpO}_{2}$ less than $80 \%$ when accompanied by symptoms and signs of severe hypoxaemia

- Sudden pallor

- Loss of coordination

- Mental confusion

- Dizziness or faintness

- Signs of respiratory distress

$\mathrm{SpO}_{2}$, peripheral capillary $\mathrm{O}_{2}$ saturation. 
Table 2 Cardiopulmonary exercise test variables

\begin{tabular}{ll}
\hline Variable & Definition \\
\hline Peak oxygen & Mean $\mathrm{VO}_{2}$ over the last $30 \mathrm{~s}$ of CPET \\
uptake $\left(\mathrm{VO}_{2 \text { peak }}\right)$ & $\begin{array}{l}\text { Reported in raw units }(\mathrm{mL}), \text { adjusted for } \\
\text { body mass }(\mathrm{mL} / \mathrm{kg} / \mathrm{min}) \text { and lean body mass } \\
\text { determined using DXA ( } \mathrm{mL} / \mathrm{kg} / \mathrm{min})\end{array}$
\end{tabular}

Ventilatory

anaerobic

threshold (VAT)
Determined using the V-slope method using the middle 5 of 7 breath data averaging.

Reported in raw units ( $\mathrm{mL})$, adjusted for body mass (mL/kg/min) and lean body mass determined using DXA (mL/kg/.min).

\section{Significance}

Traditional definition of peak aerobic fitness and limit of cardiovascular function

Indicative of cardiovascular disease severity, universal prognosticator

Abnormal when below $85 \%$ of the predicted value

Represents the point above which, further increments in work rate are increasingly sustained through anaerobic metabolism.

Objective marker of submaximal aerobic fitness/endurance. $\mathrm{A} \mathrm{VO}_{2}$ at VAT between $40 \%$ and $60 \% \mathrm{VO}_{2 \text { peak }}$ is considered normal

In conjunction with the attainment of one other marker of peak performance, RER of $>1.10$ is indicative of a 'peak' effort during CPET

Index of ventilatory efficiency representing the matching of ventilation and perfusion of the lungs and heart, respectively, as well as peripheral chemoreceptor sensitivity Slope $>34$ suggest poor prognosis

Index of ventilatory efficiency with strong correlation to

Oxygen uptake The slope relationship between the efficiency slope logarithmically transformed minute ventilation $\mathrm{VO}$ (X-axis) and $\mathrm{VO}_{2}(\mathrm{Y}$-axis) throughout the entire CPET

Reported in arbitrary units

$\mathrm{VO}_{2 \text { peak }}$

Slope $<1.4$ considered to indicate poor prognosis

High accuracy even when exercise tests are not maximal

Oxygen uptake The highest plateau in $\mathrm{VO}_{2}$ in relation to VE.

efficiency plateau Reported as the highest consecutive values (OUEP) of $\mathrm{VO}_{2}$ NE over $90 \mathrm{~s}$.

Indicates the efficiency of oxygen uptake and global

cardiovascular function

Can be used to profile severity of CHD and CHF with mean plateau values of 20-30 ( $\left.\mathrm{VO}_{2} / \mathrm{NE} \mathrm{mL} / \mathrm{L}\right)$ for CHF phenotypes Low OUEP (<65\% predicted) prognostic

Indirect measure of stroke volume response to exercise $\mathrm{O}_{2} / \mathrm{HR}$ plateau or reduction despite increases work rates, especially a lower-to-moderate work rates may indicate falling stroke volume and possible myocardial ischaemia/ myocardial wall motion abnormality.

Low $\mathrm{O}_{2}$ pulse $(<85 \%$ predicted) and early plateau/reduction in $\mathrm{O}_{2}$ pulse indicate poorer prognosis

$\mathrm{CHD}$, coronary heart disease; CHF, chronic heart failure; CPET, cardiopulmonary exercise testing; DXA, dual X-ray absorptiometry; $\mathrm{VE}$, minute ventilation; $\mathrm{VO}_{2 \text { peak, }}$ peak oxygen uptake; $\mathrm{VCO}_{2}$, carbon doxide elimination; $\mathrm{VO}_{2}$, oxygen uptake.

expiratory volume in $1 \mathrm{~s}\left(\mathrm{FEV}_{1}\right)$ and forced vital capacity (FVC) measurements. ${ }^{48} \mathrm{FEV}_{1}$, FVC and peak expiratory flow will be recorded. Maximum voluntary ventilation will be estimated using the calculation $\mathrm{FEV}_{1} \times 40 .{ }^{49-51}$

\section{DXA scan}

Body composition will be analysed using DXA (Lunar iDXA, GE Healthcare, Buckinghamshire, UK). Body composition analysis will be performed by the Lunar iDXA's integrated software. Total body mass, total body fat, compartmental body fat, lean body mass and compartmental lean body mass will be recorded for this study. Total body mass will be used for the calculation of BMI.

\section{Echocardiogram}

A trained echocardiograph technician will conduct each echocardiogram. Standard echocardiogram techniques will be used including 2D, M-mode, pulse wave Doppler to assess cardiac structure and function (systolic and diastolic). Left ventricular function will be determined from 2D echocardiography. Left ventricular function will be assessed by estimation on a catagorical scale of normal, mild, mild-to-moderate, moderate, moderate-to-severe and severe. Left ventricular ejection fraction (LVEF) will be calculated using the Simpson's formula from measurements of end-diastolic and end-systolic volumes on apical 4-chamber and 2-chamber views, following the guidelines of Schiller et al. ${ }^{52}$ Left ventricular systolic dysfunction (LVSD) will be diagnosed if LVEF is $\leq 45 \%$. When LVEF cannot be calculated, LVSD will be diagnosed were $\mathrm{LVEF} \leq 45$ or there is at least 'mild-to-moderate' impairment.

\section{Carotid intima-media thickness}

C-IMT will be measured using an automated ultrasound system (Panasonic CardioHealth Station, 
Panasonic Biomedical Sales Europe BV, Leicestershire, UK). This system has low measurement variability in healthy and cardiac populations when investigations are conducted by experienced and inexperienced operator's alike. ${ }^{345}$ C-IMT will be assessed using previously outlined methods. ${ }^{34}$ Briefly, the CHS is equipped with a broadband probe $(5-13 \mathrm{MHz})$ with a centre frequency optimised for carotid imaging. When correctly positioned over the CCA, automated integrated software locates the vessel's far wall using a region of interest tool. The CHS automatically captures a sequence of images at end-diastole by monitoring vessel distension characteristics and 'freezes' when predefined C-IMT boundary quality criteria are met. Multiple measurements taken from a $1 \mathrm{~cm}$ segment of the CCA located $1 \mathrm{~cm}$ proximally from the carotid bifurcation will be obtained. C-IMT will be measured at the right anterior $\left(150^{\circ}\right)$, lateral $\left(120^{\circ}\right)$ and posterior $\left(90^{\circ}\right)$ aspects and on the left anterior $\left(210^{\circ}\right)$, lateral $\left(230^{\circ}\right)$ and posterior $\left(270^{\circ}\right)$ aspects. Mean and maximum $(\max )$ IMT will be recorded to three decimal places. Image quality will be manually inspected and trace lines modified where required. To enhance measurement reproducibility, the probe is equipped with an accelerometer and gyroscope that tracks the angle $\left({ }^{\circ}\right)$ of insonation relative to ground. Each C-IMT measurement is recorded with the angle that the image was taken.

\section{Blood samples}

Blood samples will be drawn and placed in a refrigerated $\left(4^{\circ} \mathrm{C}\right)$ centrifuge at 3000 revolutions per minute, for $15 \mathrm{~min}$. Routine testing will include full blood cell count, total cholesterol, estimated LDL cholesterol, HDL cholesterol, Triglycerides, kidney (eGFR) and liver function tests, non-fasting glucose and NT-proBNP. Additional blood serum and plasma samples will be stored in a $-80^{\circ} \mathrm{C}$ freezer for future analysis of current and emerging biochemical markers of cardiovascular and metabolic health.

\section{Estimated all-cause mortality}

A 5-year risk of all-cause mortality will be calculated for each patient using the CALIBER 5-year prognostic risk score for stable CHD phenotypes (https://www.ucl.ac. $\mathrm{uk} /$ health-informatics/caliber). ${ }^{40}$ The CALIBER risk assessment model includes sociodemographics, CVD diagnosis and severity, CVD and non-CVD comorbidities, primary risk factors, psychosocial risk factors and plasma biomarkers.

\section{CR exercise intervention}

Patients in the TG will undergo a routine 8-week (two times weekly, 16 sessions) CR exercise programme. A physiotherapist will conduct a one-to-one assessment before each patient commences exercise training. A personal exercise prescription will be developed for each individual. Patients will be asked to self-monitor exercise intensity and be encouraged to maintain a HR corresponding to $40 \%-70 \%$ of their predicted heart rate
Table 3 Example cardiovascular and active recovery exercises

\begin{tabular}{ll}
\hline $\begin{array}{l}\text { Cardiovascular circuit } \\
\text { exercises }\end{array}$ & $\begin{array}{l}\text { Active recovery } \\
\text { exercises }\end{array}$ \\
\hline Box stepping & Arm curls \\
Static cycling & Sit to stand \\
\hline Treadmill walking & Wall press-up \\
Concept II rower & Leg curls \\
Marching on the spot & Lateral arm raises \\
Knee raises & Trunk rotation \\
\hline Half stars & \\
\hline
\end{tabular}

reserve (HRR) or an exercise 'effort' between 'light' and 'somewhat hard' (11-14) on Borg's rating of perceived exertion. ${ }^{54}$ Estimated training zones will be calculated using the Karvonen formula:

$((206-(0.7 \times$ age $))-$ resting heart rate $(-30$ if taking beta-blockers $)$.

Heart rate will be monitored with a Polar heart rate monitor. HR and RPE will be recorded at the end of each $\mathrm{CV}$ exercise station. This conforms to the recommendations of the Association of Chartered Physiotherapists in Cardiac Rehabilitation ${ }^{55}$ and the British Association of Cardiac Prevention and Rehabilitation ${ }^{56} 57$ (ie, >20-min aerobic exercise at 40\%-70\% HRR). An example list of CV and active recovery (AR) exercises are displayed in table 3.

Each exercise circuit will consist of a structured eight or nine station programme incorporating $\mathrm{CV}$ and $\mathrm{AR}$ exercises. CV exercises will initially be prescribed for approximately 1-2min duration and up-titrated for each session depending on HR and RPE responses. The target CV exercise duration for each session will be $20 \mathrm{~min}$ although $\mathrm{CV}$ exercise duration may be less than this in the first instance.

\section{Statistical analysis}

The primary endpoint for statistical analysis is the mean change in $\mathrm{VO}_{\text {2peak }}(\mathrm{mL} / \mathrm{kg} / \mathrm{min})$ from visit 1 to visit 2. For statistical purposes, visit 3 will be treated as a follow-up. This will establish the initial effect of the 8-week exercise intervention and any effects that it may have on CRF and cardiometabolic health over the 12-month study period. A main effect and an interaction effect for $\mathrm{VO}_{2 \text { peak }}$ will be investigated using a general linear model (parametric approach). The number of patients achieving a $\mathrm{VO}_{2 \text { peak }}$ improvement greater than 0.5 and 1.5 METs will also be reported. ${ }^{24}{ }^{26}$ These values correspond to improvements in CRF resulting from UK and international CR, respectively. Changes in other CRF variables will be discussed within the context of clinically meaningful thresholds (table 2) Baseline $\mathrm{VO}_{2 \text { peak }}$, age and the categorical covariate, gender will be entered as covariates in exploratory analysis. Significant differences in group characteristics identified at baseline will also be treated as covariates. Secondary outcome measures, including C-IMT, and both maximal and submaximal CRF fitness 
measures will be evaluated using the same approaches and covariates as the primary outcome analysis. Continuous measures of exercise dose will be used to predict changes to peak $\mathrm{VO}_{2 \text { peak }}$ and other CPET variables.

Data will be entered into SPSS by a single investigator who will maintain overall responsibility for data quality. The primary and secondary outcome analyses will be conducted at the conventional (two-sided) $5 \%$ alpha level. Where parametric data distribution allows, partial eta squared values will also be reported. To reduce the risk of false-positive claims, secondary analyses will be considered exploratory if non-significant results are obtained from the primary analysis. All analyses will be performed on an intention-to-treat basis. Analysis carrying the last observed values forward (baseline or 3-month outcomes) will be performed for patients lost to follow-up. A per protocol analysis will also be conducted. Patients completing at least 14 (out of 16) exercise sessions will be classed as having completed CR. No timeframe for completion will be imposed, as CR is typically extended to incorporate any missed exercise sessions. All data will be summarised and reported in accordance with the Consolidated Standards of Reporting Trials guideline. ${ }^{48}$

Power analysis, performed in G-Power ${ }^{58}$ showed that 203 patients (total) would be needed to attain statistical significance between the two groups. This was based on an estimated post-intervention between group (TG compared with CG) $\mathrm{VO}_{2 \text { peak }}$ difference of $2 \mathrm{~mL} / \mathrm{kg} / \mathrm{min}$ with a pooled $\mathrm{SD}$ of $4 \mathrm{~mL} / \mathrm{kg} / \mathrm{min} /$. A $2 \mathrm{~mL} / \mathrm{kg} / \mathrm{min}$ difference was selected based on a predicted 0.52 MET $(\mathrm{mL} / \mathrm{kg} / \mathrm{min})$ CRF increase recently reported in UK CR programmes. ${ }^{24} \mathrm{~A}$ power of $90 \%$ and a group allocation ratio of $70 \%$ TG (123 participants) to $30 \%$ CG (80 participants) with a predicted study attrition rate of $15 \%$ were applied. The assumption of uneven group sizes was made based on a local audit reporting that more patients participate in structured exercise than decline (TG 57\%; CG 43\%).

Approximately 440 patients attend the local nurse-led CR clinic each year. With a recruitment rate of $10 \%$, (44 patients per year) the study duration is estimated to be 5 years. The first patient was recruited in March 2014 and recruitment is ongoing. The study is expected to complete in March 2019. A formal interim analysis ${ }^{59}$ on the primary and secondary outcomes will be conducted when 70 patients have completed the study (one-third of the cohort required on the a priori determined sample size). A decision on trial progression will be collectively made by the research team (estimated to be January 2018). A data monitoring committee will not be used owing to the observational nature of the study.

\section{CR exercise prescription analysis}

Recent evidence ${ }^{11}$ suggests that no single exercise component within $\mathrm{CR}$ is predictive of mortality outcomes. However, reductions in both total and cardiovascular mortality were reported in trials which reported high levels of participant exercise adherence compared with those recording lower levels. ${ }^{11}$ Patients' exercise doses have also been related to long-term survival outcomes. ${ }^{60}$ Accordingly, all exercise training characteristics, including adherence to the programme, will be recorded. CV exercise duration achieved by each patient at each of their 16 CR sessions will be calculated and summed to report a total exercise training duration. To characterise exercise intensity during each exercise session, the mean of patients' HR following completion of all CV exercises for each session will be calculated. Patients' 'mean peak HR' for each exercise session will be pooled for analysis. A 'median of the mean' HR will be reported. 'Median peak HR' will expressed as a percentage of the VAT determined from visit 1 CPET and relative to HRR obtained from visit 1 CPET. A simple composite score of intensity and CV exercise duration for each training session will be calculated and summed to provide an overall 'exercise dose' for each participant. The composite score will be:

\section{Mean peak HR \\ Patients $^{\prime}$ CPET HRR $\times$ CV exercise duration .}

As an additional marker of exercise intensity the mean of a patient's RPE following completion of an exercise session will be calculated (mean RPE). As with HR, patient's RPE scores for each exercise session will be pooled for analysis.

\section{Dissemination and impact}

It is anticipated that throughout the trial, the experiences gained will be presented at national conferences and non-academic outlets such as national governing body publications. On completion, the study results will be published in peer-reviewed journals and presented at scientific meetings.

Acknowledgements We would like to acknowledge the significant contribution of Hull's cardiac rehabilitation nurses-W Summer, L Richardson and E Smith.

Contributors SN is responsible for protocol design, study approval, data collection and analysis and, presentation of findings. He was also responsible for drafting this manuscript. FN is responsible for drafting this manuscript and is involved in data collection and analysis. TG is responsible for protocol design and patient recruitment. ALC is responsible for drafting this manuscript and facilitating patient testing. SC is responsible for protocol design, study approval and drafting this manuscript. $\mathrm{LI}$ is the principal investigator and was responsible for protocol design, study approval and drafting this manuscript.

Funding This research received no specific grant from any funding agency in the public, commercial or not-for-profit sectors.

Competing interests None declared.

Patient consent Obtained.

Ethics approval Ethical approval has been obtained from the Humber Bridge NHS Research Ethics Committee-Yorkshire and the Humber (12/YH/0278). Any protocol amendments will be submitted to the committee prior to implementation.

Provenance and peer review Not commissioned; externally peer reviewed.

Open Access This is an Open Access article distributed in accordance with the Creative Commons Attribution Non Commercial (CC BY-NC 4.0) license, which permits others to distribute, remix, adapt, build upon this work non-commercially, and license their derivative works on different terms, provided the original work is properly cited and the use is non-commercial. See: http://creativecommons.org/ licenses/by-nc/4.0/

(C) Article author(s) (or their employer(s) unless otherwise stated in the text of the article) 2018. All rights reserved. No commercial use is permitted unless otherwise expressly granted. 


\section{REFERENCES}

1. Townsend N, Bhatnager P, Wilkins E, et al. Cardiovascular disease statistics 2015. 2015.

2. BACPR. Standards and core components for cardiovascular disease prevention and rehabilitation. 2017 http://www.bacpr.com.

3. Bethell H, Lewin R, Dalal H. Cardiac rehabilitation in the United Kingdom. Heart 2009;95:271-5.

4. Dalal HM, Zawada A, Jolly K, et al. Home based versus centre based cardiac rehabilitation: cochrane systematic review and metaanalysis. BMJ 2010;340:b5631.

5. Doherty P, Salman A, Furze G, et al. Does cardiac rehabilitation meet minimum standards: an observational study using UK national audit? Open Heart 2017;4:e000519.

6. JBS3 Board. Joint British Societies' consensus recommendations for the prevention of cardiovascular disease (JBS3). Heart 2014;100:ii1-67.

7. Piepoli MF, Corrà U, Adamopoulos S, et al. Secondary prevention in the clinical management of patients with cardiovascular diseases. Core components, standards and outcome measures for referral and delivery: a policy statement from the cardiac rehabilitation section of the European Association for Cardiovascular Prevention \& Rehabilitation. Endorsed by the committee for practice guidelines of the European society of cardiology. Eur J Prev Cardiol 2014;21:664-81.

8. Jolliffe JA, Rees K, Taylor RS, et al. Exercise-based rehabilitation for coronary heart disease. Cochrane Database Syst Rev 2001.CD001800.

9. Anderson L, Oldridge N, Thompson DR, et al. Exercise-Based cardiac rehabilitation for coronary heart disease: cochrane systematic review and meta-analysis. J Am Coll Cardiol 2016;67:1-12.

10. Taylor RS, Unal B, Critchley JA, et al. Mortality reductions in patients receiving exercise-based cardiac rehabilitation: how much can be attributed to cardiovascular risk factor improvements? Eur $J$ Cardiovasc Prev Rehabil 2006;13:369-74.

11. Abell B, Glasziou P, Hoffmann T. The contribution of individua exercise training components to clinical outcomes in randomised controlled trials of cardiac rehabilitation: a systematic review and meta-regression. Sports Med Open 2017;3:19.

12. van Halewijn G, Deckers J, Tay HY, et al. Lessons from contemporary trials of cardiovascular prevention and rehabilitation: A systematic review and meta-analysis. Int J Cardiol 2017;232:294-303.

13. Heran BS, Chen JM, Ebrahim S, et al. Exercise-based cardiac rehabilitation for coronary heart disease. Cochrane Database Syst Rev 2011.CD001800.

14. Bethell HJN, Turner SC, Mullee MA. Cardiac rehabilitation in the community: 11 year follow-up after a randomized controlled trial. Coronary Health Care 1999;3:183-8.

15. Carson P, Phillips R, Lloyd M, et al. Exercise after myocardial infarction: a controlled trial. 1982.

16. West RR, Jones DA, Henderson AH. Rehabilitation after myocardia infarction trial (RAMIT): multi-centre randomised controlled trial of comprehensive cardiac rehabilitation in patients following acute myocardial infarction. Heart 2012;98:637-44.

17. Ross R, Blair SN, Arena R, et al. Importance of assessing cardiorespiratory fitness in clinical practice: a case for fitness as a clinical vital sign: a scientific statement from the American Heart Association. Circulation 2016;134:e653-99. CIR. 0000000000000461

18. Martin BJ, Arena R, Haykowsky M, et al. Cardiovascular fitness and mortality after contemporary cardiac rehabilitation. Mayo Clin Proc 2013;88:455-63.

19. Mandic S, Myers J, Oliveira RB, et al. Characterizing differences in mortality at the low end of the fitness spectrum in individuals with cardiovascular disease. Eur J Cardiovasc Prev Rehabil 2010;17:289-95.

20. Vanhees L, Fagard R, Thijs L, et al. Prognostic value of traininginduced change in peak exercise capacity in patients with myocardial infarcts and patients with coronary bypass surgery. Am J Cardiol 1995;76:1014-9.

21. Barons MJ, Turner S, Parsons N, et al. Fitness predicts long-term survival after a cardiovascular event: a prospective cohort study. BMJ Open 2015;5:e007772.

22. Taylor C, Tsakirides C, Moxon J, et al. Submaximal fitness and mortality risk reduction in coronary heart disease: a retrospective cohort study of community-based exercise rehabilitation. BMJ Open 2016;6:e011125.

23. Dugmore LD, Tipson RJ, Phillips $\mathrm{MH}$, et al. Changes in cardiorespiratory fitness, psychological wellbeing, quality of life, and vocational status following a 12 month cardiac exercise rehabilitation programme. Heart 1999;81:359-66.
24. Ingle L, Carroll S. Cardiac rehabilitation and exercise training. Heart 2013:99:1298.1-1298.

25. Sandercock G, Cardoso F, Almodhy M. Cardiorespiratory fitness changes in patients receiving comprehensive outpatient cardiac rehabilitation in the UK: a multicentre study. Heart 2013;99:1298-9.

26. Sandercock G, Hurtado V, Cardoso F. Changes in cardiorespiratory fitness in cardiac rehabilitation patients: a meta-analysis. Int $J$ Cardiol 2013;167:894-902

27. Houchen-Wolloff L, Boyce S, Singh S. The minimum clinically important improvement in the incremental shuttle walk test following cardiac rehabilitation. Eur J Prev Cardiol 2015;22:972-8.

28. Nichols S, Gleadall-Siddall DO, Antony R, et al. Estimated peak functional capacity: an accurate method for assessing change in peak oxygen consumption after cardiac rehabilitation? Clin Physiol Funct Imaging 2017 (Epub ahead of print 30 Aug 2017).

29. Sandercock GR, Cardoso F, Almodhy M, et al. Cardiorespiratory fitness changes in patients receiving comprehensive outpatient cardiac rehabilitation in the UK: a multicentre study. Heart 2013;99:785-90.

30. Boden WE, Franklin BA, Wenger NK. Physical activity and structured exercise for patients with stable ischemic heart disease. JAMA 2013;309:143-4.

31. Kachur S, Chongthammakun V, Lavie CJ, et al. Impact of cardiac rehabilitation and exercise training programs in coronary heart disease. Prog Cardiovasc Dis 2017;60:103-14.

32. Hambrecht R, Walther C, Möbius-Winkler S, et al. Percutaneous coronary angioplasty compared with exercise training in patients with stable coronary artery disease: a randomized trial. Circulation 2004;109:1371-8.

33. Pignoli $P$, Tremoli E, Poli A, et al. Intimal plus medial thickness of the arterial wall: a direct measurement with ultrasound imaging. Circulation 1986;74:1399-406.

34. Nichols S, Milner M, Meijer R, et al. Variability of automated carotid intima-media thickness measurements by novice operators. Clin Physiol Funct Imaging 2016;36:25-32.

35. Amato $M$, Montorsi $P$, Ravani $A$, et al. Carotid intima-media thickness by B-mode ultrasound as surrogate of coronary atherosclerosis: correlation with quantitative coronary angiography and coronary intravascular ultrasound findings. Eur Heart $J$ 2007;28:2094-101.

36. Feairheller DL, Diaz KM, Kashem MA, et al. Effects of moderate aerobic exercise training on vascular health and blood pressure in African Americans. J Clin Hypertens 2014;16:504-10.

37. Kim SH, Lee SJ, Kang ES, et al. Effects of lifestyle modification on metabolic parameters and carotid intima-media thickness in patients with type 2 diabetes mellitus. Metabolism 2006;55:1053-9.

38. Byrkjeland R, Stensæth $\mathrm{KH}$, Anderssen S, et al. Effects of exercise training on carotid intima-media thickness in patients with type 2 diabetes and coronary artery disease. Influence of carotid plaques. Cardiovasc Diabetol 2016;15:13.

39. Brodie D, Bethell H, Breen S. Cardiac rehabilitation in England: a detailed national survey. Eur J Cardiovasc Prev Rehabil 2006;13:122-8

40. Rapsomaniki E, Shah A, Perel P, et al. Prognostic models for stable coronary artery disease based on electronic health record cohort of 102023 patients. Eur Heart J 2014;35:844-52.

41. Department of Health. Department of HealthDepartment of Health's commissioning pack on cardiac rehabilitation. In: ed. 2010.

42. American Thoracic SocietyAmerican College of Chest Physicians. ATS/ACCP Statement on cardiopulmonary exercise testing. Am J Respir Crit Care Med 2003;167:211.

43. ACSM. ACSM's Guidelines for exercise testing and prescription. 9th ed. Philadelphia: Wolters Kluwer/Lippincott Williams \& Wilkins Health, 2013.

44. Taylor C, Nichols S, Ingle L. A clinician's guide to cardiopulmonary exercise testing 1: an introduction. Br J Hosp Med 2015;76:192-5.

45. Nichols S, Taylor C, Ingle L. A clinician's guide to cardiopulmonary exercise testing 2: test interpretation. Br J Hosp Med 2015;76:281-9.

46. Balady GJ, Arena R, Sietsema K, et al. Clinician's guide to cardiopulmonary exercise testing in adults: a scientific statement from the American Heart Association. Circulation 2010;122:191-225.

47. Bruce RA, Kusumi F, Hosmer D. Maximal oxygen intake and nomographic assessment of functional aerobic impairment in cardiovascular disease. Am Heart J 1973;85:546-62.

48. Miller MR, Hankinson J, Brusasco V, et al. Standardisation of spirometry. Eur Respir J 2005;26:319-38.

49. Hansen JE, Sue DY, Wasserman K. Predicted values for clinica exercise testing. Am Rev Respir Dis 1984;129:S49-55.

50. Campbell SC. A comparison of the maximum voluntary ventilation with the forced expiratory volume in one second: an assessment of subject cooperation. J Occup Med 1982;24:531-3. 
51. Blackie SP, Fairbarn MS, McElvaney NG, et al. Normal values and ranges for ventilation and breathing pattern at maximal exercise. Chest 1991;100:136-42.

52. Schiller NB, Shah PM, Crawford M, et al. Recommendations for quantitation of the left ventricle by two-dimensional echocardiography. American Society of Echocardiography Committee on Standards, Subcommittee on Quantitation of Two-Dimensional Echocardiograms. J Am Soc Echocardiogr 1989;2:358-67.

53. Vanoli D, Wiklund U, Lindqvist $P$, et al. Successful novice's training in obtaining accurate assessment of carotid IMT using an automated ultrasound system. Eur Heart J Cardiovasc Imaging 2014;15:637-42.

54. Borg GA. Psychophysical bases of perceived exertion. Med Sci Sports Exerc 1982;14:377-81.
55. ACPICR. Standards for physical activity and exercise in the cardiovascular population. 3rd ed. Association of Chartered Physiotherapists in Cardiac Rehabilitation, 2015.

56. BACPR. Standards and core components for cardiovascular disease prevention and rehabilitation. 2012. http://www.bacpr.com/ resources/

57. BACR. British association for cardiac rehabilitation: exercise instructor training module. 4th edn. Leeds: Human Kinetics, 2006.

58. Faul F, Erdfelder E, Buchner A, et al. Statistical power analyses using G*Power 3.1: tests for correlation and regression analyses. Behav Res Methods 2009;41:1149-60.

59. Herson J, Wittes J. The use of interim analysis for sample size adjustment. Drug Inf J 1993;27:753-60.

60. Taylor C, Tsakirides C, Moxon J, et al. Exercise dose and all-cause mortality within extended cardiac rehabilitation: a cohort study. Open Heart 2017;4:e000623. 